\title{
EGRESSOS DE INTERNAÇÃO PSIQUIÁTRICA ACOMPANHADOS NA REDE DE SERVIÇOS DE SAÚDE
}

\section{PATIENTS DISCHARGED FROM PSYCHIATRIC HOSPITAL CARE FOLLOWED IN THE HEALTH SERVICE NETWORK}

\section{EGRESOS DE INTERNACIÓN PSIQUIÁTRICA ACOMPANHADOS EM LOS SERVICIOS DE LA RED DE SALUD}

\author{
Sueli Aparecida de Castro ${ }^{1}$, Antônia Regina Ferreira Furegato ${ }^{2}$, Jair Lício Ferreira Santos ${ }^{3}$
}

\begin{abstract}
RESUMO
Objetivo: Acompanhar e avaliar a adesão do portador de transtorno mental aos tratamentos, após sua alta hospitalar. Método: Estudo descritivo transversal baseado em dados primários e secundários. Análise das variáveis sociodemográficas e clínicas informadas e registradas nos retornos aos serviços, coletadas durante 21 meses. Resultados: Dos 875 egressos, 71 atenderam aos critérios de inclusão do estudo. Maioria branca, entre 40 e 49 anos, sexo masculino, solteiros, sem companheiro, nível fundamental incompleto, internações anteriores e prevalência dos diagnósticos Esquizofrenia e Transtorno afetivo bipolar. O estado mental prevalente na alta foi calmo, associado a vários diagnósticos e sem proposta terapêutica para o pós-alta. Maioria compareceu aos retornos agendados, com pouca adesão aos tratamentos oferecidos, segundo o Teste Morisky-Green. Acolhimentos realizados principalmente pela equipe de enfermagem. $O$ atendimento das equipes não está centrado na reabilitação psicossocial. Conclusão: O portador de transtorno mental comparece aos serviços de saúde mental, porém não se observam resultados significativos na sua reabilitação.
\end{abstract}

Descritores: Serviços de saúde mental; Enfermagem psiquiátrica; Reabilitação; Equipe de assistência ao paciente.

\begin{abstract}
Objective: To monitor and evaluate the adherence of patients with mental disorders to treatments after their hospital discharge. Method: Descriptive cross-sectional study based on secondary dataand primary. Analysis of sociodemographic and clinical variables reported and recorded in returns services were collected for 21 months and analyzed. Results: From the 875 patients discharge, 71 patients, who met the inclusion criteria, were selected for the study. Most were Caucasian, aged from 40 to 49 years old, male, single, without a partner/spouse, with incomplete primary school. Most of them were previously hospitalized and the diagnoses Schizophrenia and Bipolar affective disorderwere prevalent. The most common mental state at the time of release was calm, associated with other diagnoses butit has no therapeutic proposal for post-discharge. Most attended scheduled their appointments, however, did not adhere to the medication regimen, according to the Morisky-Green test. The welcome care for these patients into the services is mainly performed by nursing staff. The team's attention is

\footnotetext{
${ }^{1}$ Sueli Aparecida de Castro, Enfermagem, Doutora em Ciências, Escola de Enfermagem de Ribeirão Preto-USP, Telefones: (16) 39748631 (16)988654524, E-mail: castrossueli@ gmail.com

2 Antônia Regina Ferreira Furegato, Enfermagem, Professor Titular do Departamento de Enfermagem psiquiátrica e Ciências Humanas da Escola de Enfermagem de Ribeirão Preto-USP, Telefones:(16)3602-0535 (16) 9761-8010, E-mail: furegato@eerp.usp.br

${ }^{3}$ Jair Lício Ferreira Santos, Demógrafo, Professor Titular do Departamento de Medicina Social, Faculdade de Medicina de Ribeirão Preto USP, Telefones (16) 36022443 (16)3315 3070, E-mail: jairlfs@ fmrp.usp.br
} 
not focused on psychosocial rehabilitation.Conclusion: The mental patients appeared to mental health services but do not observe significant results in their rehabilitation.

Descriptors: Mental health services; Psychiatric nursing; Rehabilitation; Patient care team.

\section{RESUMEN}

Objetivo: Supervisar y evaluar la adherencia de los pacientes mentales para el tratamiento después de su alta hospitalaria. Método: Estudio descriptivo, transversal basado en datos secundarios (registros) y primarios (entrevistas). Análisis de las variables sociodemográficas y clínicas descritas y registradas en los servicios de devoluciones se recogieron durante 21 meses y se analizó. Resultados: De los 875 egresos, 71atendieron a los criterios de inclusión del estudio. La mayoría era blanca, entre los 40 y 49 años, sexo masculino, solteros, sin compañeros, nivel fundamental incompleto, internaciones anteriores y con prevalencia de los diagnósticos Esquizofrenia y Trastorno afectivo bipolar.El estado de ánimo predominante en alta, estaba en calma, asociado con múltiples diagnósticos y sin propuesta terapéutica para la post alta. La mayoría procuróa las citas programadas, con poca adherencia a los tratamientos que se ofreció, según la prueba de Morisky-Green. Los cuidados se llevan a cabo principalmente por el personal de enfermería. La atención del equipo no se centra en la rehabilitación psicosocial. Conclusión: Los pacientes mentales procuran a los servicios de salud mental, pero no se observan resultados significativos en su rehabilitación.

Palabras clave: Servicios de salud mental; Enfermería Psiquiátrica; Rehabilitación; Equipo de asistencia al paciente.

\section{INTRODUÇÃO}

A carga global das doenças decorrentes de transtornos mentais continua aumentando gradativamente no mundo e calcula-se que as doenças crônicas e os distúrbios mentais representam 59\% do total de óbitos no mundo. Presume-se que esse percentual atingirá $60 \%$ até o ano 2020 e as maiores incidências serão de doenças cardíacas, acidente vascular cerebral e câncer. ${ }^{1}$

A atenção básica, através da Estratégia de Saúde da Família, configura um campo de práticas e de produção de novos modos de cuidado em saúde mental. Atualmente, a articulação entre as equipes da saúde mental e atenção básica é um desafio a ser enfrentado para a melhoria da assistência prestada e a ampliação do acesso da população aos serviços, com garantia de continuidade de atenção. ${ }^{2}$

A legislação brasileira em saúde mental considera a reabilitação psicossocial, uma estratégia potente que pode contribuir para a expansão do modelo de Atenção Psicossocial. ${ }^{3}$ Entretanto, avaliações têm apontado que o processo de trabalho das equipes dos serviços comunitários, ainda, segue a lógica do atendimento ambulatorial, distante das diretrizes do modelo psicossocial que contempla a subjetividade, as relações interpessoais e que incentiva a construção de um novo processo de trabalho, através da valorização do sentido que o outro atribui à sua experiência. Segundo a 
percepção dos próprios usuários desses serviços, a inclusão está relacionada ao trabalho, educação, renda, poder contratual e ser aceito na sua diferença. ${ }^{4}$

As consequências da assistência ineficiente podem ser observadas através do fenômeno da reinternação psiquiátrica ${ }^{5}$, que parece confirmar falhas nas intervenções vigentes.

Diante desse cenário, é necessário aprimorar o conhecimento acerca da doença e da assistência a ela relacionada, o acesso aos serviços, a qualidade do atendimento, bem como a avaliação da efetividade dos dispositivos de atenção à saúde mental como reguladores das internações psiquiatricas e da garantia de autonomia do portador de trasntorno mental.

Portanto, este estudo objetivou acompanhar o egresso da internação psiquiátrica na rede de serviços de saúde para identificar o acesso aos serviços, o atendimento recebido, assim como comparar essas informações com os dados sociodemográficos e clínicos dos sujeitos.

\section{MÉTODO}

Trata-se de estudo descritivo transversal baseado em dados primários e secundários. Os dados primários foram obtidos através de entrevistas realizadas com o portador de transtorno mental. Em relação aos dados secundários referentes à alta hospitalar, assim como retornos e acompanhamento dos pacientes nos serviços extra-hospitalares, as informações foram levantadas respectivamente, nos boletins de alta hospitalar e nos prontuários dos serviços de saúde mental extrahospitalares.

Das 875 altas realizadas no hospital psiquiátrico regional, apenas $44 \%$ residiam em Ribeirão Preto, sendo que 71 preencheram os critérios estabelecidos no estudo (maiores de 18 anos), residentes em Ribeirão Preto, sem diagnóstico de dependência de substâncias psicoativas F10-F19. Dos 377 de Ribeirão Preto, 19\% saíram sem agendamento e foram encaminhados para a rede particular ou mudaram-se no período.

$\mathrm{O}$ estudo foi realizado entre maio de 2013 e janeiro de 2015. O levantamento das altas ocorreu de maio de 2013 a junho de 2014, para acompanhar o egresso num prazo suficiente para preencher todos os registros da pesquisa. $\mathrm{O}$ acompanhamento de todos os sujeitos estendeu-se até janeiro de 2015.

Os locais da pesquisa foram $\mathrm{o}$ Hospital Santa Tereza de Ribeirão Preto (HST-RP), Centro de Atenção Psicossocial II Dr. Cláudio Roberto C. Rodrigues (CAPS II), Ambulatório Regional de Saúde Mental- Distrito Central - Dr. Nelson Okano (ASM-DC), Núcleo de Saúde Mental (NSM) e Ambulatório de 
Saúde Mental da Unidade Básica de Saúde- Dr. Ítalo Baruffi (ASM-UBS).

Os instrumentos utilizados para a coleta dos dados foram: 1) Roteiro para obtenção de dados secundários da internação (HST-RP): Dados coletados no Serviço de Arquivo do Hospital Santa Tereza de Ribeirão Preto, através do Boletim de alta hospitalar. O roteiro foi composto por 18 itens, com questões de identificação dos sujeitos, dados sociodemográficos e clínicos sobre o portador de transtorno mental de sua admissão na internação (sexo, idade, etnia, estado civil, arranjo domiciliar, número de internações, data da última admissão) e da alta hospitalar (data, diagnóstico, condições mentais, psicofármacos prescritos, proposta terapêutica, local e data do agendamento); 2) Roteiro para obtenção dos dados nos serviços extrahospitalares (SEH): Dados coletados nos prontuários dos serviços referenciados da rede ARSM, CAPS II e NSM. Esse roteiro foi estruturado com 23 itens para obter informações referentes ao serviço, comparecimento (data agendada, antes do dia), motivo alegado para o comparecimento antes do agendamento, acolhimento, acompanhante, data do próximo agendamento e dados clínicos do portador de transtorno mental, como o diagnóstico médico, estado mental, psicofármacos prescritos, atendimentos de outros profissionais, intercorrência no intervalo entre a alta e o primeiro retorno (data, local), acolhimento, acompanhante; 3) Roteiro para Entrevista (Serviços extra hospitalares): As entrevistas foram sempre realizadas em uma sala reservada, nos serviços selecionados para a pesquisa e as informações foram registradas em formulário semi estruturado. Duraram de 15 a 30 minutos, levantando informações sociodemográficas e clínicas, como a idade, ocupação, estado civil/arranjo domiciliar, escolaridade, doença, medicamentos prescritos, quem procura no serviço quando tem problema, importância dos medicamentos, esquecimento e cuidados com os horários de tomar seu remédio, apoio e ajuda com os remédios e retorno. Além disso, o roteiro continha questões abertas sobre os serviços: profissionais interessados nas suas dificuldades pessoais, motivação para o tratamento, procura de serviço fora da consulta agendada, avaliação do serviço (facilidade para marcar consultas, tempo de espera, atendimento pela equipe, dias/horário de funcionamento, localização, infraestrutura), apoios, preconceito e sugestões para melhorar o atendimento das suas necessidades (questão descritiva); 4) Teste de Adesão ao Tratamento de Morisky-Green: Esse teste é composto por quatro perguntas que avaliaram o grau de adesão ao tratamento 
medicamentoso e foi aplicado ao término da entrevista. A partir dos dados obtidos, os pacientes foram classificados no grupo de alto grau de adesão, quando todas as respostas forem negativas; e baixo grau de adesão, quando pelo menos uma das respostas for afirmativa $\left.{ }^{6,7} ; 5\right)$ Roteiro para obtenção de dados no segundo retorno nos serviços extra-hospitalares: Informações coletadas nos prontuários do respectivo serviço extra hospitalar. Trata-se de um instrumento de acompanhamento dos retornos que contempla as seguintes questões: Compareceu no segundo retorno? Compareceu fora do dia agendado? Alterou o diagnóstico? Alterou o medicamento?

Todos os roteiros utilizados para coleta dos dados primários e secundários foram construídos pelas pesquisadoras, com base na experiência profissional e na literatura sobre o tema.

Intencionalmente, são apresentados, no presente texto, os dados quantitativos, obtidos das fontes secundárias, relacionados à caracterização sociodemográfica e clinica, bem como as propostas terapêuticas para os egressos de internação psiquiátrica, para análise dos dados referentes aos comparecimentos aos serviços de saúde, nos retornos agendados, além do teste de adesão medicamentoso. Os dados qualitativos, obtidos nas entrevistas serão apresentados em outro artigo.

O projeto foi aprovado por Comitê de Ética, atendendo normas do CEP 196/96 (CEP-EERP/USP218/2013), autorizado por Comitê de Pesquisa do HST-RP, da Secretaria da Saúde de Ribeirão Preto e do NSM. Os participantes foram informados sobre a natureza do estudo, objetivos e procedimentos. Os sujeitos que concordaram em participar da pesquisa assinaram o TCLE.

Os procedimentos de análise foram baseados em análise estatística relacionando a adesão ao tratamento às variáveis sociodemográficas, clínicas e do acompanhamento nos serviços. As categorias utilizadas para classificação dos dados foram a caracterização dos sujeitos, acompanhamento dos sujeitos pós-alta hospitalar e o teste Morisk e Green sobre a adesão do sujeito ao tratamento medicamentoso. A discussão teve como suporte a literatura sobre o tema.

\section{RESULTADOS}

\section{Caracterização dos sujeitos}

Dos 71 sujeitos do estudo, 36 $(50,7 \%)$ são do sexo masculino. As faixas etárias com maior frequência foram: 40 a 49 anos $=31 \% ; 30$ a 39 anos $=23,9 \% ; 50$ aos 59 anos $=19,7 \%$. Ao analisar sexo e grupo etário observa-se que há associação estatística (Fisher=0,003), sendo a idade 
média das mulheres superior à média dos homens.O estado civil prevalente é de solteiros $(52,1 \%)$, dos quais $22(61,1 \%)$ são homens e $15(42,8 \%)$ são mulheres e 80,3\% dos sujeitos vivem só.

Ao analisar o grau de escolaridade observa-se que $35,2 \%$ (25) têm o nível fundamental incompleto e 19,7\% (14) médio incompleto. Verificou-se que 19,7\% (14) dos prontuários não tinham informação sobre o grau de escolaridade do paciente.Os registros na tabela 1 mostram também que $66,2 \%$ dos pacientes são inativos.

Tabela 1 - Caracterização sociodemográfica dos sujeitos com altas psiquiátricas, após internação hospitalar, segundo registro no Boletim de alta.

\begin{tabular}{|c|c|c|c|c|c|c|c|}
\hline \multirow[t]{2}{*}{ Variáveis } & \multirow[t]{2}{*}{ Idade (anos) } & \multicolumn{2}{|c|}{$\begin{array}{c}\text { Mulheres } \\
\qquad(\mathbf{n}=35)\end{array}$} & \multicolumn{2}{|c|}{$\begin{array}{l}\text { Homens } \\
\qquad(\mathrm{n}=\mathbf{3 6})\end{array}$} & \multicolumn{2}{|c|}{$\begin{array}{l}\text { Totais } \\
(n=71)\end{array}$} \\
\hline & & $\mathbf{N}$ & $\%$ & $\mathbf{N}$ & $\%$ & $\mathbf{N}$ & $\%$ \\
\hline \multirow{5}{*}{ Grupo etário } & Até 29 & 2 & 5.7 & 8 & 22.2 & 10 & 14.1 \\
\hline & 30 a 39 & 6 & 17.1 & 11 & 30.6 & 17 & 23.9 \\
\hline & 40 a 49 & 12 & 34.3 & 10 & 27.8 & 22 & 31 \\
\hline & 50 a 59 & 9 & 25.7 & 5 & 14 & 14 & 19.7 \\
\hline & $60 e+$ & 6 & 17.1 & 2 & 5.6 & 8 & 11.3 \\
\hline \multirow{4}{*}{ Etnia } & Branca & 20 & 57.1 & 24 & 66.7 & 44 & 62 \\
\hline & Negra & 4 & 11.4 & 1 & 2.8 & 5 & 7 \\
\hline & Parda & 8 & 22.9 & 7 & 19.4 & 15 & 21.1 \\
\hline & Sem informação & 3 & 8.6 & 4 & 11.1 & 7 & 9.9 \\
\hline \multirow{5}{*}{ Estado civil } & Solteiro & 15 & 42.9 & 22 & 61.1 & 37 & 52.1 \\
\hline & Casado & 6 & 17.1 & 8 & 22.2 & 14 & 19.7 \\
\hline & Separado & 9 & 25.7 & 4 & 11.1 & 13 & 18.3 \\
\hline & Viúvo & 5 & 14.3 & 1 & 2.8 & 6 & 8.5 \\
\hline & Sem informação & & & 1 & 2.8 & 1 & 1.4 \\
\hline \multirow{4}{*}{$\begin{array}{l}\text { Arranjo } \\
\text { Domiciliar }\end{array}$} & Vive só & 28 & 80 & 29 & 80.6 & 57 & 80.3 \\
\hline & & & & & & & \\
\hline & Com companheiro & 7 & 20 & 6 & 16.7 & 13 & 18.3 \\
\hline & Sem informação & & & 1 & 2.8 & 1 & 1.4 \\
\hline \multirow{8}{*}{ Escolaridade } & $\begin{array}{l}\text { Analfabeto } \\
\text { Fundamental }\end{array}$ & 2 & 33,3 & 4 & 66,7 & 6 & 8,5 \\
\hline & incompleto & 12 & 48 & 13 & 52 & 25 & 35,2 \\
\hline & Fundamental & 2 & 66,7 & 1 & 33,3 & 3 & 4,2 \\
\hline & Médio completo & 2 & 40 & 3 & 60 & 5 & 7 \\
\hline & Médio incompleto & 10 & 71,4 & 4 & 28,6 & 14 & 19,7 \\
\hline & Superior completo & 2 & 66,7 & 1 & 33,3 & 3 & 4,2 \\
\hline & Superior incompleto & & & 1 & 100 & 1 & 1,4 \\
\hline & Sem informação & 5 & 35,7 & 9 & 64,3 & 14 & 19,7 \\
\hline Atividade & Inativo & 24 & 68,6 & 23 & 63,9 & 47 & 66,2 \\
\hline
\end{tabular}




\begin{tabular}{llcccccc} 
profissional & Ativo & 1 & 2,9 & 3 & 8,3 & 4 & 5,6 \\
& Afastado & 1 & 2,9 & 5 & 13,9 & 6 & 8,5 \\
& Sem Informação & 9 & 25,7 & 5 & 13,9 & 14 & 19,7 \\
Totais & & $\mathbf{3 5}$ & $\mathbf{4 9 , 3}$ & $\mathbf{3 6}$ & $\mathbf{5 0 , 7}$ & $\mathbf{7 1}$ & $\mathbf{1 0 0}$ \\
\hline
\end{tabular}

A proporção de homens sem internações anteriores no HST-RP é menor que a proporção das mulheres, sendo 9 (25\%) e $14 \quad(40 \%)$, respectivamente; 7 (20\%) homens e 7 $(19,4)$ mulheres têm duas internações anteriores; com três e mais internações somam-se $38 \%$, sendo $15(41,7 \%)$ do sexo masculino e $12(34,3 \%)$ do sexo feminino. Não se observou associações significativas (Fisher=0,438).

Os diagnósticos prevalentes na alta foram F20-F29 (23:32,4\%) e F30-f39 (22:31\%) e a quantidade de psicofármacos prescritos variou de 1 a 7 , sendo que $38 \%$ (27), $26,8 \%$ (19), $16,9 \%$ (12) e $11,3 \%$ (8) saíram com a prescrição de $3,4,2$ e 5 psicofármacos, respectivamente.

Os agendamentos dos sujeitos nos serviços da rede ocorreram na seguinte proporção: CAPSII $\quad(39,4 \%)$, NSM (36,6\%), ASM-UBS $(22,6 \%)$ e ASM-DC $(1,4 \%)$.

$\mathrm{Na}$ associação entre sexo e proposta terapêutica para acompanhamento extrahospitalar verifica-se que $52,1 \%$ dos sujeitos não tiveram qualquer proposta terapêutica e para 40,9\%, a indicação terapêutica limitou-se apenas ao "seguimento ambulatorial". A recomendação de psicoterapia e atendimento multiprofissional corresponde a somente $1,4 \%$ e $4,2 \%$, respectivamente. $\mathrm{O}$ teste exato de Fisher não confirma associação entre as duas variáveis (Fisher=0,443\%). Entretanto, os dados permitem constatar que não há continuidade terapêutica, o que prejudica a longitudinalidade desejada para o acompanhamento do portador de transtorno mental na rede de atenção à saúde.

Os resultados, a seguir, correspondem aos dados dos retornos do sujeito ao serviço comunitário, após a alta hospitalar, que foram registradas no prontuário do paciente em cada um dos serviços para onde foram encaminhados.

Verificou-se que 84,5\% (60) dos sujeitos compareceram ao primeiro retorno agendado no serviço extra-hospitalar, após sua alta hospitalar. Os faltosos estão distribuídos proporcionalmente entre os sexos, sendo $16,7 \%$ do sexo masculino e $14,3 \%$ do sexo feminino.

Verifica-se na Tabela 2, a distribuição do acolhimento realizado pela 
equipe dos serviços comunitários aos portadores de transtorno mental, por ocasião do comparecimento ao primeiro retorno na data e, também, fora da data agendada. Observa-se que a equipe de enfermagem foi responsável por mais de
$62 \%$ de todos os acolhimentos no primeiro retorno. Vale registrar que a equipe de enfermagem também acolheu mais de $60 \%$ dos sujeitos que compareceram fora da data agendada, no terceiro retorno.

Tabela 2 - Distribuição dos pacientes conforme comparecimento na data agendada para o primeiro retorno, em relação ao profissional que realizou seu acolhimento.

\begin{tabular}{lcccccc}
\hline & \multicolumn{7}{c}{ Comparecimento na data agendada } \\
Acolhimento & \multicolumn{2}{c}{ Sim } & \multicolumn{2}{c}{ Não } & \multicolumn{2}{c}{ Total } \\
& $\mathbf{N}$ & $\%$ & $\mathbf{N}$ & $\mathbf{\%}$ & $\mathbf{N}$ & $\%$ \\
\hline $\begin{array}{l}\text { Assistente social, médico, psicólogo e terapeuta } \\
\text { ocupacional }\end{array}$ & 9 & 90 & 1 & 10 & 10 & 15,1 \\
Auxiliar e técnico de enfermagem & 19 & 90,5 & 2 & 10 & 21 & 31,8 \\
Enfermeiro & 18 & 90 & 2 & 20 & 20 & 30,3 \\
Outros & 14 & 93,3 & 1 & 7 & 15 & 22,7 \\
Totais & $\mathbf{6 0}$ & $\mathbf{9 0 , 9}$ & $\mathbf{6}$ & $\mathbf{1 0}$ & $\mathbf{6 6}$ & $\mathbf{1 0 0}$ \\
\hline
\end{tabular}

A proporção de comparecimentos ao segundo retorno agendado foi acima de $80 \%$ em todas as faixas etárias, exceto entre os mais jovens até 29 anos (70\%). Registrou-se também maior presença de transferências para outros serviços entre homens, mais jovens.
Conforme Tabela 3, observa-se que no segundo retorno há maior proporção de pacientes com diagnósticos F20 a F29 (32\%), seguidos por F30 a F39 (31\%), dentre os 71 sujeitos da pesquisa.

Tabela 3 - Registro de comparecimentos dos pacientes ao segundo retorno e transferências, de acordo com o local de seguimento.

\begin{tabular}{|c|c|c|c|c|c|c|c|c|c|c|}
\hline \multirow{3}{*}{ Serviço } & \multicolumn{10}{|c|}{ Comparecimento ao segundo retorno agendado } \\
\hline & \multicolumn{2}{|c|}{ Sim } & \multicolumn{2}{|c|}{ Não } & \multicolumn{2}{|c|}{ Transferência } & \multicolumn{2}{|c|}{ Outro } & \multicolumn{2}{|c|}{ Total } \\
\hline & $\mathbf{N}$ & $\%$ & $\mathbf{N}$ & $\%$ & $\mathbf{N}$ & $\%$ & $\mathbf{N}$ & $\%$ & $\mathbf{N}$ & $\%$ \\
\hline ASM-UBS & 12 & 80 & 1 & 6,7 & 2 & 13,3 & & & 15 & 21,1 \\
\hline ASM-DC & & & & & 1 & & & & 1 & 1,4 \\
\hline CAPS II & 25 & 92,6 & 2 & 7,4 & & & & & 27 & 38 \\
\hline Núcleo & 19 & 67,9 & 4 & 14,3 & 2 & 7,1 & 3 & 10,7 & 28 & 39,4 \\
\hline
\end{tabular}


Na Tabela 4, encontram-se os dados correspondentes ao cruzamento das variáveis "comparecimento ao terceiro retorno agendado" e "grupo etário", distribuídos nas diferentes faixas etárias onde se observa que somente nos maiores de 60 compareceram todos. Quanto à ausência ao retorno agendado, os números mostram que as proporções das ausências foram maiores na faixa dos 30 aos $39 \operatorname{anos}(4: 40 \%)$ e dos 50 aos $59 \operatorname{anos}(3: 30 \%)$. Em relação aos diagnósticos, a prevalência foi de F20$29(17: 37 \%)$ e o acolhimento foi realizado, na maioria das vezes, pela enfermagem.

Tabela 4 - Distribuição das altas psiquiátricas, segundo o comparecimento do paciente ao terceiro retorno agendado, em relação ao seu grupo etário.

\begin{tabular}{|c|c|c|c|c|c|c|}
\hline \multirow{3}{*}{ Grupo etário } & \multicolumn{6}{|c|}{ Comparecimento ao retorno agendado } \\
\hline & \multirow{2}{*}{$\begin{array}{r}\operatorname{Sim} \\
\mathbf{N}\end{array}$} & & \multicolumn{2}{|l|}{ Não } & \multicolumn{2}{|l|}{ Total } \\
\hline & & $\%$ & $\mathbf{N}$ & $\%$ & $\mathbf{N}$ & $\%$ \\
\hline Até 29 anos & 5 & 71 & 2 & 29 & 7 & 14 \\
\hline 30 a 39 anos & 9 & 69 & 4 & 31 & 13 & 27 \\
\hline 40 a 49 anos & 13 & 93 & 1 & 7,1 & 14 & 29 \\
\hline 50 a 59 anos & 6 & 67 & 3 & 33 & 9 & 18 \\
\hline 60 anos e + & 6 & 100 & & & 6 & 12 \\
\hline Totais & 39 & 80 & 10 & 20 & 49 & 100 \\
\hline
\end{tabular}

O Teste de Adesão ao Tratamento de Morisky-Green indicou que não houve associação significativa entre categoria de adesão e acolhimento (Fisher=0,062). Em relação aos serviços, o Teste revelou associação significativa de adesão ao tratamento medicamentoso no ASM-UBS com 27,3\% (Fisher=0,027), enquanto que o NSM não alcançou adesão em 17 (94,4\%) e o CAPS II teve 100 \% de não adesão das pessoas em seguimento.

\section{DISCUSSÃO}

Nessa pesquisa encontrou-se 857 altas do hospital psiquiátrico regional HST-RP, no período de maio de 2014 a junho de 2015.

Das 71 altas, acompanhadas pelo estudo, quase $30 \%$ foram encaminhadas ao 
CAPS II e, aproximadamente, dois terços, foram agendadas em serviços ambulatoriais de saúde mental, os quais proporcionam menor oferta de intervenções reabilitadoras.

A amostra final foi constituída principalmente por brancos, com ensino fundamental incompleto, inativos, prevalência de solteiros do sexo masculino. Em relação ao arranjo domiciliar, verificou-se alto percentual dos que vivem só. Este perfil está em consonância com pesquisas anteriores, realizadas no mesmo serviço e com outros serviços no país. ${ }^{8,9}$

A idade média das mulheres em alta hospitalar psiquiátrica é maior do que a idade média dos homens. A prevalência do sexo feminino está na faixa dos 40 aos 49 anos e a maior proporção do sexo masculino na faixa dos 30 aos39 anos.

Essa pesquisa mostrou que as mulheres consomem mais psicofármacos do que homens e a grande maioria ingere 3 ou mais psicofármacos. Dessa forma, o medicamento se constitui no principal recurso disponível para o tratamento dos portadores de transtorno mental, tornandoos dependentes de medicamentos. ${ }^{10,11,12}$

Verifica-se a priorização do tratamento medicamentoso em detrimento às intervenções psicossociais, o que revela a fragilidade das intervenções realizadas pelas equipes, intra e extra-hospitalares, pois os medicamentos por si só não resgatam os benefícios sociais e restringem-se a estabilização do quadro psíquico, para que as ações reabilitadoras sejam desenvolvidas.

Evidenciou-se que $68 \%$ dos sujeitos, apresentaram reinternações, proporção que está acima de estudo anterior no mesmo serviço $^{13}$, podendo ser explicado pela exclusão de alguns diagnósticos como uso de álcool e outras drogas e pelo não seguimento dos sujeitos em acompanhamento no ASM-DC. Entretanto, esse percentual está de acordo com estudos semelhantes. $^{14,15}$

Chamou a atenção, neste estudo, a falta de registro de propostas terapêuticas para o pós-alta e a falta de operacionalização do Projeto Terapêutico Singular (PTS). Não se encontrou no boletim de alta e na ficha de contra referência das pessoas de alta hospitalar nenhuma descrição de intervenções psicossociais. Nos poucos registros havia informações somente sobre os medicamentos prescritos ou recomendação de "seguimento ambulatorial". Lamentavelmente, esses resultados estão em conformidade com outros serviços no Brasil. $^{16,17}$

A maior proporção dos sujeitos não apresentou adesão ao tratamento medicamentoso, segundo o teste de adesão de Morisk-Green. Esse resultado incentiva a reflexão sobre os objetivos da internação 
psiquiátrica e a valorização que se dá ao acompanhamento do portador de transtorno mental na rede de serviços comunitários, cuja finalidade, além da extinção ou minimização dos sintomas é a de desenvolver ações que possam contribuir para melhor adesão ao tratamento medicamentoso juntamente com sua inserção psicossocial.

A associação significativa entre a categoria de adesão e diagnóstico na alta está de acordo com os estudos que afirmam a dificuldade de adesão ao tratamento entre os pacientes portadores de transtorno de humor e transtornos esquizofrênicos. A dificuldade de adesão ao tratamento é referenciada por outros estudiosos. ${ }^{18}$

O ASM-UBS foi o serviço que alcançou maior adesão ao tratamento; todavia, esse resultado é limitado, pois esse serviço foi o que obteve o menor número de atendimentos, em relação aos demais. Além disso, trata-se de um serviço de atendimento ambulatorial, sem ações de reabilitação psicossocial.

A análise do comparecimento ao retorno agendado, segundo o diagnóstico, mostra que mais da metade dos sujeitos, portadores de transtornos esquizofrênicos e transtornos do humor, compareceram ao retorno, conforme o agendamento nos três retornos, sendo que o comparecimento fora do retorno agendado é maior entre os portadores de esquizofrenia. Porém, ao longo do acompanhamento, observa-se redução da busca pelo serviço.

Esses dados mostram que os portadores de transtornos mentais têm motivação para manter o tratamento psiquiátrico, todavia parece que ao longo do tratamento não recebem estímulos necessários para a continuidade do tratamento, o que sugere que somente o comparecimento ao serviço não está associado à adesão ao tratamento medicamentoso, pois depende ainda do tipo da assistência, acompanhamento e estabelecimento de ví nculo entre equipe, paciente e família. ${ }^{5}$

O não comparecimento ao retorno agendado pode representar a ineficiência das estratégias de atendimento nos serviços pesquisados, com ações desvinculadas da Rede de Atenção a Saúde (RAS) e prejudicar ações que poderiam contribuir para o portador de transtorno mental corresponsabilizar-se pelo seu próprio tratamento e para potencializar o envolvimento dos familiares.

Os resultados dos comparecimentos aos retornos agendados, segundo o sexo, mostram que as mulheres compareceram mais que os homens, ao longo do acompanhamento, o que pode implicar em maior vulnerabilidade dos homens para internação psiquiátrica, cujos índices são maiores no sexo masculino. ${ }^{19}$ Ao analisar o número de internações anteriores observa- 
se que, a distribuição do número internações psiquiátricas variou de 1-3 e mais e esteve em alta proporção, de acordo com outros estudos encontrados na literatura.

O acompanhamento dos sujeitos durante os retornos permitiu observar que sua ausência à consulta médica foi de no máximo $1 / 4$ do total. As demais ausências se deram por motivos conhecidos e justificados, o que significa que grande parcela dos sujeitos compareceu aos retornos médicos agendados. Este resultado pode significar que somente o comparecimento aos retornos médicos não é suficiente para a adesão ao tratamento. Além disso, dois sujeitos não compareceram ao terceiro pelo motivo de nova reinternação psiquiátrica. Esse evento pode mostrar que apesar de terem comparecido em todos os retornos anteriores pode-se concluir que deve haver outros fatores importantes que influenciam fortemente essa adesão.

Chamou a atenção, também, a maior proporção do acolhimento ter sido realizada pelos profissionais da enfermagem (enfermeiros, auxiliares e técnicos), o que pode ser justificado pela permanência dessa equipe em todo o período de funcionamento dos serviços e principalmente, pelo estabelecimento de vínculo por realizarem prioritariamente as suas ações junto ao paciente. A formação profissional do enfermeiro sustenta $\mathrm{O}$ cuidado como foco principal. ${ }^{20}$ Esse dado reafirma a importância de investimentos na educação desses profissionais.

Devido à complexidade das necessidades dos portadores de transtorno mental, considera-se que as equipes da atenção básica podem ter maior participação, serem mais resolutivas, identificarem riscos e vulnerabilidades, necessidades e demandas de saúde ${ }^{2}$, pois esse nível de atenção organiza o fluxo dos usuários entre os pontos de atenção da RAS, assim como em outras estruturas das redes de saúde. ${ }^{20}$

As limitações desse estudo foram a impossibilidade de acompanhar metade dos egressos da internação psiquiátrica, encaminhados para Ribeirão Preto devido a não autorização para a realização da pesquisa pela gerencia do ASM-DC, o encerramento do funcionamento do NSM, na fase final do estudo, sem nenhum planejamento prévio, o que causou enorme dificuldade para o preenchimento do último instrumento, pois os usuários ficaram sem referência de serviço para continuar o tratamento e o longo período de tempo entre o agendamento das consultas, que impactou na extensão do período de acompanhamento dos sujeitos da pesquisa (20 meses).

A fragmentação da continuidade do plano terapêutico, observado neste 
estudo, leva a supor que o sujeito poderá ter prejuízos para seu tratamento, implicando em perda da qualidade da atenção. Este tema merece outros estudos futuros.

\section{CONCLUSÕES}

A presente pesquisa permitiu constatar que tanto nos registros do Boletim de Alta Hospitalar assim como nos registros dos serviços extrahospitalares da rede, o atendimento pelas equipes multiprofissionais não foi acompanhado de Projeto Terapêutico Singular.

A maioria dos sujeitos recebeu alta hospitalar sem proposta terapêutica para o pós-alta ou, simplesmente, com prescrição de seguimento ambulatorial.

Essa conduta evidencia que a organização de serviços permanece voltada para o atendimento das condições agudas, sem vínculo com o acompanhamento do sujeito nos serviços da rede básica de saúde, o que pode contribuir para a descontinuidade da assistência integral.

Verificou-se priorização do tratamento medicamentoso tanto pelas equipes, intra como extra-hospitalares, em detrimento das intervenções psicossociais, o que revela a fragilidade das intervenções.

\section{REFERÊNCIAS}

1-Mendes E. As redes de atenção à saúde. Brasília, DF: Organização Pan -

Americana de Saúde; 2011.

2-Aosani TR, Nunes KG. A saúde mental na atenção básica: a percepção dos profissionais de saúde. Rev Psicol Saúde [Internet]. 2013 [citado em 17 out 2017]; 5(2):71-80. Disponível em: http://pepsic.bvsalud.org/scielo.php?script =sci_arttext\&pid=S2177093X2013000200002\&lng=pt

3- Gruska V, Dimenstein M. Reabilitação psicossocial e acompanhamento terapêutico. Psicol Clin. 2015; 27(1):10122.

4-Salles MM, Barros S. Representações sociais de usuários de um Centro de Atenção Psicossocial e pessoas de sua rede sobre doença mental e inclusão social. Saúde Soc. 2013; 22(4):1059-71. 5-Machado S, Santos MA. O tratamento extra-hospitalar em saúde mental na perspectiva do paciente reinternado. Psicol Estud. 2013; 18(4):701-12.

6-Morisky DE, Levini DM, Green LW, Smith CR. Health education program effects on the management of hypertension in the elderly. Arch Intern Med. 1982; 142 (10):1835-8.

7-Morisky DE, Green LW, Levini DM. Concurrent and predicitive validity of a self-reported measure of medication adherence. Med Care. 1986; 24(1):67-74. 8-Silva T1, Maftum MA, Kalinke LP, Mathias TAF, Ferreira ACZ, Capistrano FC. Perfil sociodemográfico, clinico e de internação de pacientes em tratamento na unidade psiquiátrica de um hospital geral. Cogitare Enferm. jan./mar 2015;

20(1):112-20.

9-Tabeleão VP, Tomasi E, Quevedo LA. Sobrecarga de familiares de pessoas com transtorno psíquico: níveis e fatores associados. Rev Psiquiatr Clin. 2014; 41 (3):63-6.

10-Urgell CV, Monne SB, Vega CF, Esquius NP. Estudio de utilización de psicofármacos em atención primaria. Aten Prim. [Internet]. 2005 [citado em 12 mar 2016]; 36(5):239-45. Disponívelem: 
http://www.elsevier.es/es-revista-atencionprimaria-27-articulo-estudio-utilizacionpsicofarmacos-atencion-primaria13079144

11-Rocha BS; Werlang MC.

Psicofármacos na Estratégia Saúde da Família: perfil de utilização, acesso e estratégias para a promoção do uso racional. Ciênc Saúde Colet. 2013; 18 (11):3291-300.

12-Gudes TG, Moura ERF, Almeida PC. Particularidades do planejamento familiar de mulheres portadoras de transtorno mental. Rev Latinoam Enferm. 2009; 17 (5):639-44.

13-Machado V, Santos MA. O tratamento extra-hospitalar em saúde mental na perspectiva do paciente reinternado. Psicol Estud. 2013; 18 (4):701-12.

14-Bezerra CG, Dimeinstein M.

Acompanhamento terapêutico na proposta de alta-assistida implementada em hospital psiquiátrico: relato de uma experiência. Psicol Clín. 2009; 21(1):1532.

15-Ramos DKR, Guimarães J. Novos serviços de saúde mental e o fenômeno da porta giratória no Rio Grande do Norte. REME Rev Min Enferm. 2013; 17(2):4405.

16-Silva EP, Melo FABP, Sousa MM, Gouveia RA, Tenório AA, Cabral AFF, et al. Projeto terapêutico singular como estratégia de prática da multiprofissionalidade nas ações de saúde singular. Rev Bras Ciênc Saúde. 2013; 17 (2):197-202.

17-Hori AA, Nascimento AF. O Projeto Terapêutico Singular e as práticas de saúde mental nos Núcleos de Apoio à Saúde da Família (NASF) em Guarulhos (SP), Brasil, Ciênc Saúde Colet. 2014; 19 (8):3561-71.

18-Uchida S, Hiraoka S, Namiki N. Development of gummi drugs of aripiprazole as hospital formulations. Chem Pharm Bull. 2015; 63(5):354-60. 19- Silva TL, Mariluci AM, Kalinke LP, Mathias TAF, Ferreira ACZ, Capistrano FC. Perfil sociodemográfico e clínico dos pacientes em tratamento na unidade psiquiátrica de um hospital geral. Cogitare Enferm. 2015; 20(1):112-20.

20-Santana SS, Fontenelle T, Magalhães LM. Assistência de enfermagem prestada aos pacientes em tratamento hemodialítico nas unidades de nefrologia. Rev Cient ITPAC. 2013; 6(3):1-11.

RECEBIDO: $19 / 03 / 2017$

APROVADO: $25 / 04 / 2018$

PUBLICADO: 07/2018 